\title{
Effects of food restriction and social cues on sexual maturation and growth in male musk shrews (Suncus murinus)
}

\author{
N. L. Wayne*, G. N. Wade $\ddagger$ and E. F. Rissman $\dagger$ \\ Departments of ${ }^{*}$ Biology and $\dagger P$ sychology, University of Virginia, Charlottesville, VA 22901, USA; \\ and $\ddagger$ Department of Psychology and Neuroscience and Behavior Program, University of \\ Massachusetts, Amherst, MA 01003, USA
}

\begin{abstract}
Summary. In the first experiment, the effects of food availability on growth and reproduction were assessed by placing male musk shrews on 3 different feeding schedules, i.e. fed ad libitum, fed $50 \%$ of the amount eaten by the ad-libitum controls or fed $25 \%$ of the amount eaten by the ad-libitum controls. After 25 days, growth and sexual maturation were significantly retarded in both of the food-restricted groups compared to the control group. In the second experiment, the combined effects of food availability and social cues were investigated in juvenile males that were either fed ad libitum (during the day) or placed on a restricted diet known to inhibit growth and reproduction. Half of these males were either housed alone or with an adult female (separated from female during the day by a wire barrier, but in full contact at night). The ad-libitum-fed males living with a female ate more food, gained more body weight and were more sexually mature than ad-libitum-fed animals living alone. Furthermore, males in the two food-restricted groups had lower body weights and were sexually immature compared with males in both of the ad-libitum-fed groups. Finally, there were little or no differences in growth and reproduction between animals in the two food-restricted groups. These results suggest that food availability is an important environmental regulator of the timing of sexual maturation, and that stimulatory social cues cannot override the inhibitory effects of an inadequate diet.
\end{abstract}

Keywords: growth; musk shrew; nutrition; puberty; reproduction; social cues

\section{Introduction}

The timing and occurrence of reproduction is subject to regulation by a variety of environmental influences. Most of our information on external control of breeding comes from work on animals living in the temperate zone where there are large seasonal changes in the environment. Conversely, little is known about reproductive processes in tropical mammals, and yet they too are subjected to environmental fluctuations. Changes in local external variables (e.g. food availability, social cues from conspecifics) may have profound influences on the timing of sexual maturation in tropical mammals (Bronson, 1989).

The work presented in this paper investigates the effects of two environmental variables, food availability and the presence of an adult female, on growth and sexual maturation in the male musk shrew (Suncus murinus: Order Insectivora). These shrews are insectivorous and inhibit tropical and subtropical regions throughout Asia. This animal is considered to be an opportunistic breeder since pregnant females and fertile males have been captured in the wild throughout the year (Harrison, 1955; Barbehenn, 1962; Louch et al., 1966; Beg et al., 1986), although small seasonal peaks in reproductive activity have been documented in some areas (Harrison, 1955; Barbehenn, 1962; Beg 
et al., 1986). These peaks in reproduction are correlated with seasonal changes in rainfall. For instance, on Guam Island, reproductive activity is greatest during the transition from the dry to rainy season; conversely, breeding is at its lowest period during the transition from the rainy to the dry season (Barbehenn, 1962). As a consequence of changes in rainfall patterns, there may also be changes in insect emergence and hence fluctuations in food availability.

Recent studies in the male musk shrew show that social cues emanating from an adult female have stimulatory effects on growth and sexual maturation (Rissman et al., 1990; Wayne \& Rissman, 1990), while exposure to short daylengths has inhibitory effects (Rissman et al., 1987; Wayne \& Rissman, 1990). Additional findings suggest that the juvenile male shrew integrates conflicting information from these two environmental cues. Weanling males raised in short days in the presence of a female showed greater sexual maturity than those raised in short days without a female. Furthermore, males raised under short days with a female were equivalent in terms of maturity to males raised in long days without a female, and less mature than those raised in long days in the presence of a female (Wayne \& Rissman, 1990). Based on these previous findings, we proposed that the presence of an adult female could at least partly override inhibitory effects of food restriction on sexual maturation in the male musk shrew.

\section{Materials and Methods}

General. The animals used in these studies were born and raised in our colony at the University of Virginia. The original stock descended from animals trapped in the early $1960 \mathrm{~s}$ on Guam Island at a latitiude of $13^{\circ} \mathrm{N}$ latitude. Musk shrews were kept on a light-dark cycle of $14 \mathrm{~h}$ light:10 h dark (lights on 07:00 h EST) at $23 \pm 1^{\circ} \mathrm{C}$. Water was provided ad libitum and food (Purina cat chow) was provided as described below. Isolated males were housed in plastic cages $(28 \times 17 \times 12 \mathrm{~cm})$, while animals housed with females were kept in a split cage $(38 \times 18 \times 11 \mathrm{~cm}$; a single wire-mesh barrier divided the cage in half; dimension of holes in the screen was $0.25 \mathrm{~cm}^{2}$ ). All animals were weaned at 20 days of age and were sexually naive. Males were assigned to treatment groups based on body weight; group allocation was random, but done in a manner that yielded equivalent average initial weights among groups.

The level of reproductive activity was assessed by plasma androgen concentrations, as well as the relative weights of various androgen-sensitive target tissues (one flank pad, the seminal vesicle-prostate complex, one ampulla, one testis). After 25 days, animals were anaesthetized with halothane (2-bromo-2-chloro-1,1,1-trifluorethane; Halocarbon Labs Inc., Hackensack, NJ, USA) and a single blood sample was taken via cardiac puncture between 08:00 and 11: $00 \mathrm{~h}$. Blood was centrifuged and plasma was collected and stored at $-20^{\circ} \mathrm{C}$ until assayed. Following blood collection, animals were rapidly killed by cervical dislocation and the androgen-sensitive target tissues were dissected and weighed. Sperm numbers were calculated from one testis (Exp. 1; Kirton et al., 1967)

Androgen assay. Two $100-\mu \mathrm{l}$ volumes of plasma from each sample were extracted in $2 \mathrm{ml}$ ether. The ether fraction was dried and measured for total androgen content by radioimmunoassay. The antiserum (S250; generously provided by $\mathrm{Dr}$ G. Niswender) cross-reacts with several androgens in addition to testosterone: $5 \alpha$-dihydrotestosterone $(69 \%)$; $3 \alpha$-androstanediol (14\%); 3 $\beta$-androstanediol (22\%) (Gay \& Kerlan, 1978). This assay has been described and validated elsewhere for use with musk shrew plasma (Rissman et al., 1990). All samples were run within a single assay for each of the two experiments. The intra-assay coefficient of variation $(\mathrm{CV})$ for standard sera containing $1.97,0 \cdot 17$ and $0 \cdot 10 \mathrm{ng} / \mathrm{ml}$ averaged $4 \cdot 3 \%$ for Exp. 1. The intra-assay CV for standard sera containing $2 \cdot 01,0 \cdot 10$ and $0 \cdot 06 \mathrm{ng} / \mathrm{ml}$ averaged 3.4\% for Exp. 2. The limits of detection (2 standard deviations from the buffer controls; for $100 \mu l$ serum) were 0.05 and $0.02 \mathrm{ng} / \mathrm{ml}$ for Exps 1 and 2, respectively. Recoveries of tritiated testosterone measured after extraction were 93.6 and $95.8 \%$ for Exps $\mathrm{I}$ and 2, respectively. Hormone values were not corrected for recovery.

Experiment 1. The purpose of this study was to determine the effects of food availability on growth and reproduction in the juvenile male musk shrew. At weaning, males $(\mathrm{N}=17)$ were divided into 3 groups: fed ad libitum (adlibitum group); fed $50 \%$ of the amount eaten by the ad-libitum controls ( $50 \%$ group); fed $25 \%$ of the amount eaten by the ad-libitum controls (25\% group). Each animal in the food-restricted groups was paired to an animal in the adlibitum group. The daily food intake of each ad-libitum animal was recorded and the paired partners in the $50 \%$ and $25 \%$ groups were fed the following day based on this food-intake information. Daily food consumption from animals in the ad-libitum group was determined by weighing the amount of chow left-over (in food dish and scattered about in bedding) and subtracting this value from a pre-weighed quantity of food given to the animals on the previous day. Animals in the $50 \%$ and $25 \%$ groups ate all food presented to them. Body weights were recorded every $2-6$ days. After 25 days of treatment, animals were necropsied for determination of level of sexual maturity.

Experiment 2. To test the hypothesis that the stimulatory effects of an adult female would override inhibitory effects of food restriction on growth and sexual maturation in the male musk shrew, weanling males $(\mathrm{N}=40)$ were divided into 4 groups. Half the males were fed an ad-libitum diet and the other half were restricted to $2 \cdot 5 \mathrm{~g}$ chow/day. 
Each of these 2 groups was either housed alone or with an adult female ( $>60$ days of age and sexually experienced). Based on the findings from Exp. 1, it was known that $2.5 \mathrm{~g}$ chow/day (approximate daily food consumption of 50\% group) inhibited both growth and reproduction as compared with ad-libitum feeding. To avoid food restricting the females, the experiment was designed such that females and males could be fed separately yet interact with each other. To do this, males were housed with females in a split cage and separated from them for $10-12 \mathrm{~h}$ during the light period (between 08:00 and 20:00 h EST) by a wire-mesh barrier. Males were fed a known quantity of food during the separation period (females were fed ad libitum). At the end of separation, all food was removed from both sides of the cages. Food in the males' side of the cage was weighed to determine the amount eaten (food-restricted animals ate all of their food allotment during the light period). The barrier was then removed, permitting direct contact between the pairs during the dark period (when shrews are most active). Body weights were recorded every 5 days. After 25 days, males were necropsied for determination of level of sexual maturity.

Analysis of body composition. Body composition was analysed in Exp. 2 in 7 animals/group and was measured as described by Bartness \& Wade (1984). Briefly, eviscerated shrews were weighed, then dried and finely homogenized in a blender. Lipid was extracted with petroleum ether and the remaining tissue was weighed and termed fat-free dry weight (FFDW).

Analysis of data. The weights of the androgen-sensitive target tissues were analysed using analysis of covariance with body weight being the covariate. This procedure eliminates any influences of body weight from the analysis. To determine effects of treatment, one-way or two-way analyses of variance were applied where appropriate, followed by a Student-Newman-Keuls multiple range test or Scheffé's multiple comparison procedure. Body composition data were analysed in terms of total content, as well as in terms of the percentage of carcass weight. Because the relationship between reproductive organs and body weight is not a simple proportional one, the values presented are for raw, unadjusted tissue weights. A significant alpha of $P<0.05$ was used.

\section{Results}

\section{Experiment 1: effects of food restriction on growth and sexual maturation}

Food restriction had a profound effect on the physiology of the male, reducing body weight (Fig. 1) and inhibiting reproductive maturation (Fig. 2). By the end of the study, body weight of the ad-libitum-control group was greater than that of the $50 \%$ group $(P<0.01)$, and body weights of both of these groups were greater than that of the $25 \%$ group $(P<0.01$ for each comparison). A similar pattern was seen with the seminal vesicle-prostate complex, ampulla and flank pad in which tissue weights were greatest in the ad-libitum group, significantly lower in the $50 \%$ group and least in the $25 \%$ group. The ad-libitum group had greater concentrations of plasma androgen, heavier testes and more spermatozoa/testis than did either of the food-restricted groups; however, there were no significant differences in these measurements between the 2 food-restricted groups.

\section{Experiment 2}

In males fed ad libitum, the presence of an adult female stimulated food consumption, growth and reproduction compared with animals living alone; however, in food-restricted males, the presence of a female had little or no effect. Food consumption (Fig. 3a) and body weight (Fig. 3b) were greatest in the ad-libitum + female group, intermediate in the ad-libitum alone group, and least in the 2 food-restricted groups by the end of the study ( $P<0.01$ for each comparison). There was no significant difference in body weight between the food restricted + female and the food restricted alone groups. A similar pattern was observed for total body content of water, lipid and FFDW; however, when these values were taken as a percentage of carcass weight, there were no significant differences among the groups (Fig. 4). Furthermore, the weights of the seminal vesicleprostate complex, flank pad and ampulla were greatest in the ad-libitum + female group, intermediate in the ad-libitum alone group, and least in the 2 food-restricted groups (Fig. 5). Only the seminal vesicle-prostate complex showed a significant difference in tissue weight between the 2 food-restricted groups (presence of a female having a slight stimulatory effect). In addition, circulating concentrations of androgen were greater in the ad-libitum + female group than all other groups (Fig. 5). There were no significant differences in testis weights among the groups. 


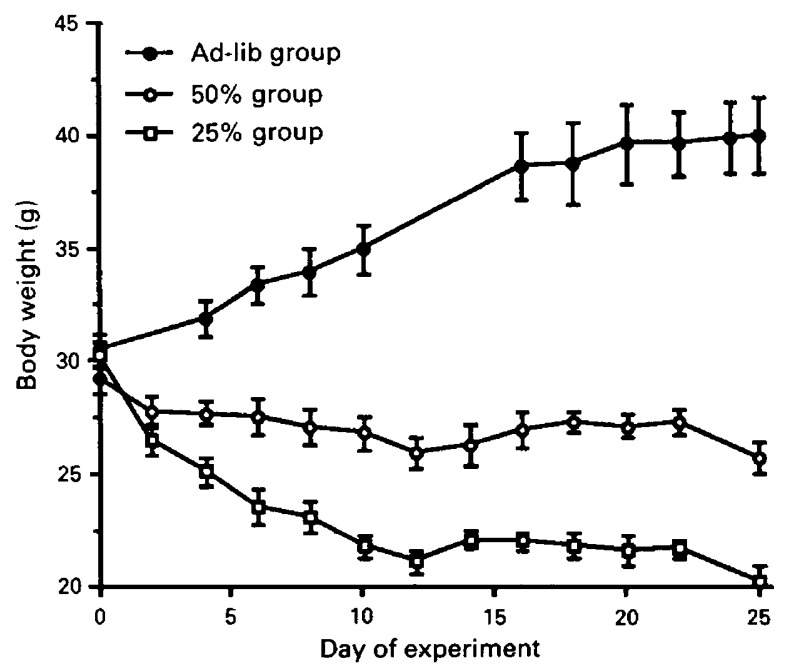

Fig. 1. Values (mean \pm s.e.m. for 5-6 animals/group) for body weights of musk shrews in Exp. 1. Animals were weaned on Day 0.
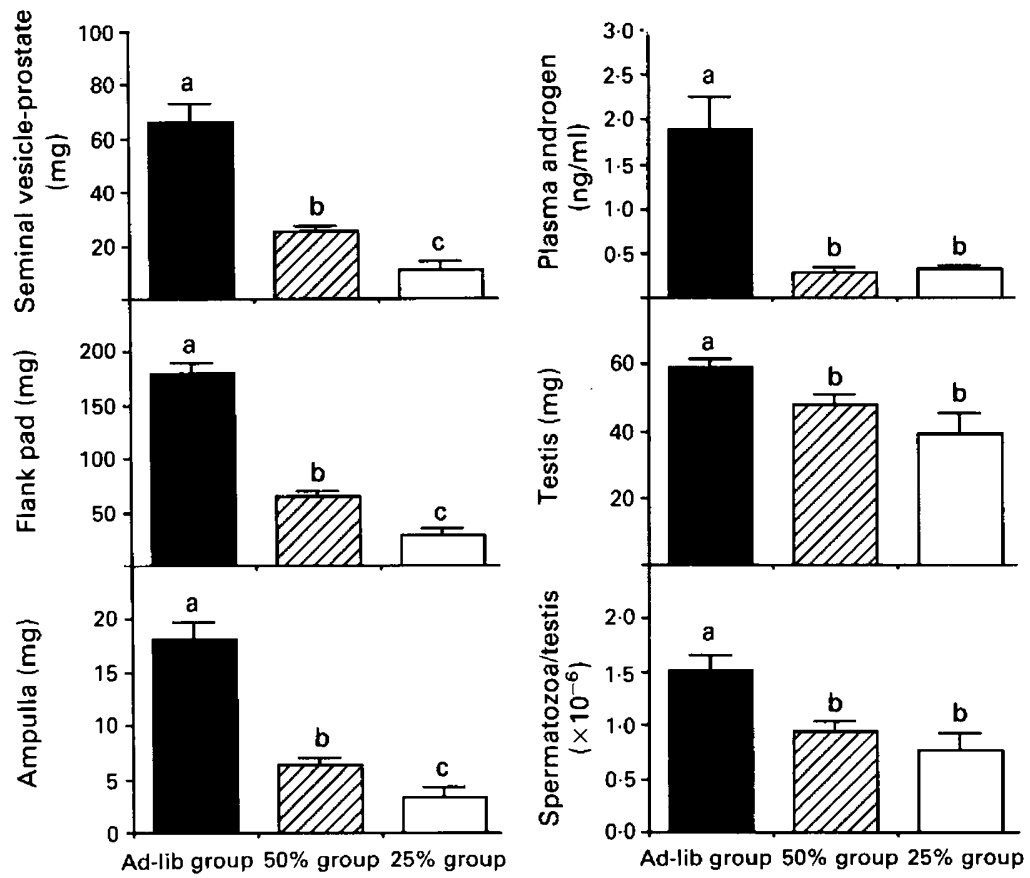

Fig. 2. Values (mean \pm s.e.m. for 5-6 animals/group) for androgen-sensitive target tissues, number of spermatozoa/testis and plasma androgen concentrations of musk shrews in Exp. 1 that were fed ad libitum (Ad-lib group), fed $50 \%$ of the amount eaten by the ad-libitum controls ( $50 \%$ group) and fed $25 \%$ of the amount eaten by the ad-libitum controls ( $25 \%$ group). Values with different letters indicate values significantly different from each other $(P<0.05)$. 

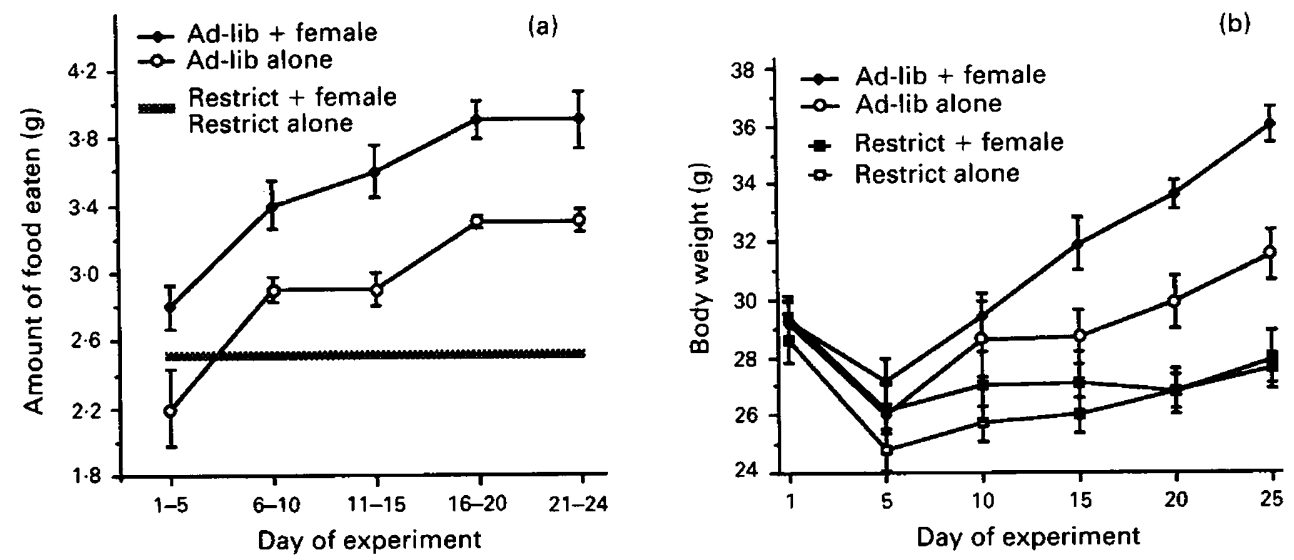

Fig. 3. Values for (a) daily food consumption and (b) body weight for musk shrews in Exp. 2. All food-restricted males were fed $2 \cdot 5 \mathrm{~g}$ food/day as indicated by a shaded line. Values are mean \pm s.e.m. for 10 animals/group. Animals were weaned on Day 0 .

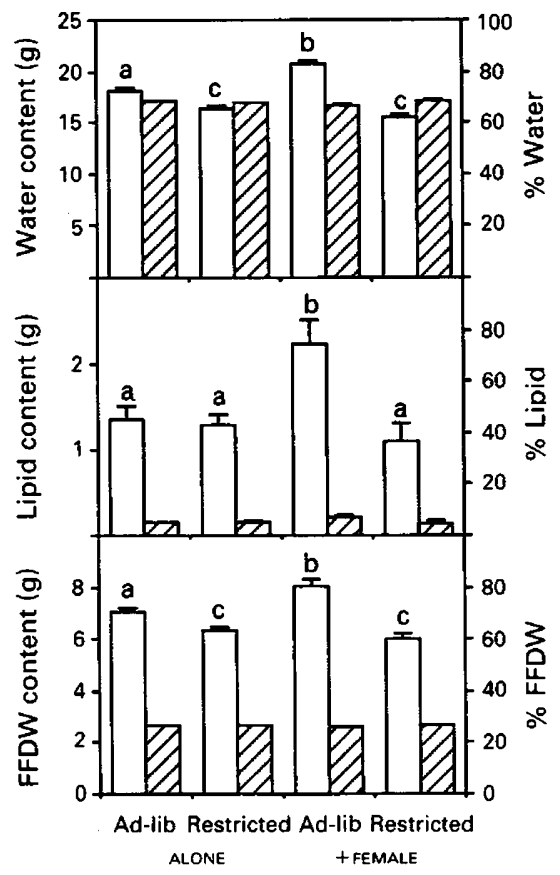

Fig. 4. Values for body composition of musk shrews in Exp. 2 are shown as the mean \pm s.e.m. for 7 animals/group. Total body content of water, lipid and FFDW are shown in the open histograms; percentages of carcass weight of water, lipid and FFDW are shown in the hatched histograms. Values with different letters indicate values significantly different from each other $(P<0.05)$.

Males fed ad libitum and living alone in Exp. 2 had lower body and reproductive organ weights than those fed ad libitum (and also living alone) in Exp. 1. This discrepency was due to differences in feeding schedules between the two experiments; animals in Exp. 2 had access to food for 10-12 h during the light period whereas those in Exp. 1 had access to food $24 \mathrm{~h} /$ day (see 'Materials and 


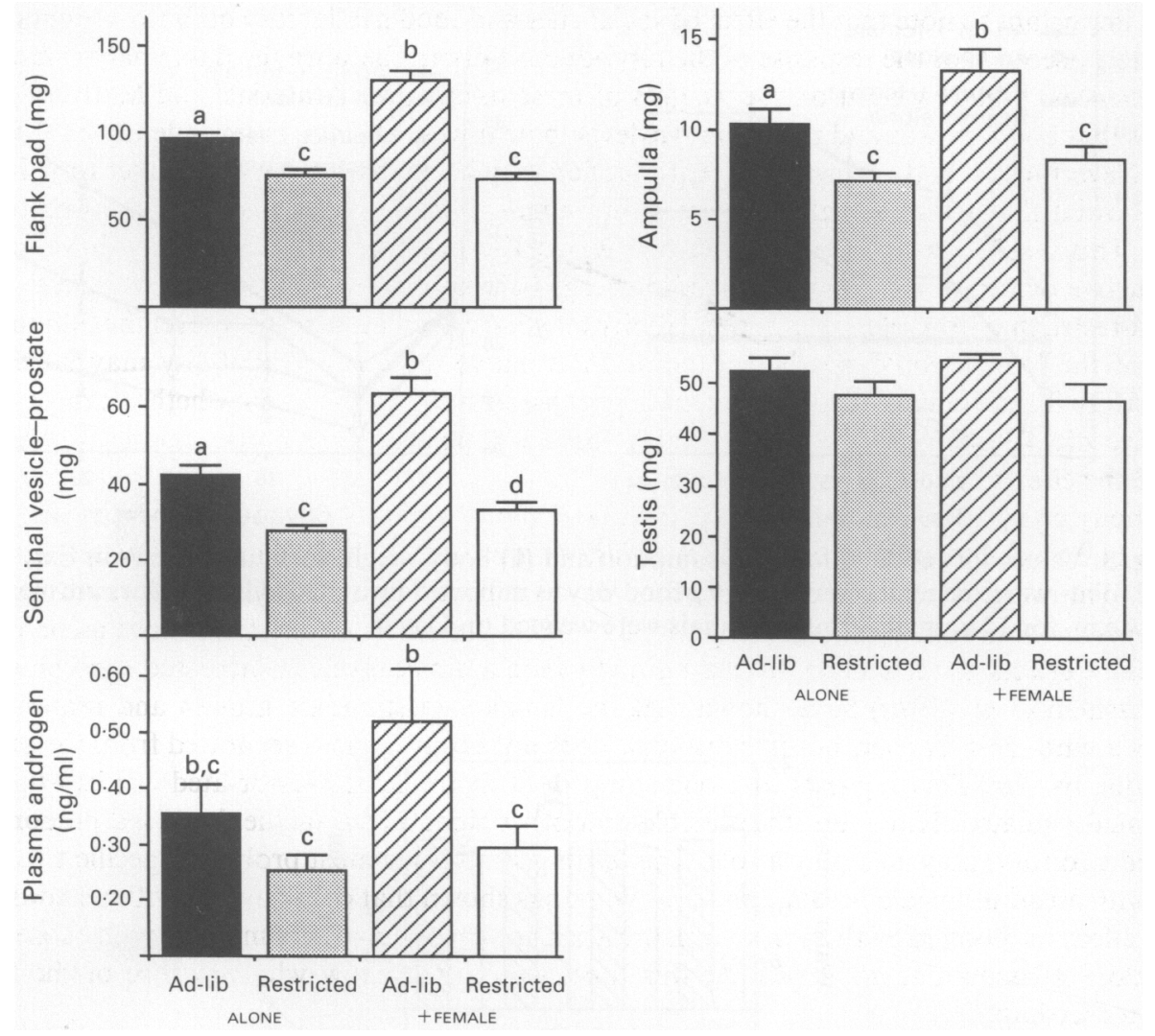

Fig. 5. Values (mean \pm s.e.m. for 10 animals/group) for androgen-sensitive target tissues and plasma androgen concentrations in musk shrews in Exp. 2. Values with different letters indicate values significantly different from each other $(P<0.05)$.

Methods'). Apparently, animals fed ad libitum during the day ate less food and therefore gained less weight than their counterparts fed ad libitum round the clock.

\section{Discussion}

The results of the present studies show that food restriction has a pronounced inhibitory effect on sexual maturation in the male musk shrew. In Exp. 1, restricting food consumption to $50 \%$ and $25 \%$ of that eaten by control animals fed ad libitum resulted in a $35 \%$ and a $50 \%$ reduction in final body weight compared with controls. Both levels of food restriction resulted in dramatic suppression of reproductive maturation, and in some reproductive parameters (weights of the seminal vesicle-prostate complex, flank pad and ampulla), tissue growth was influenced by the amount of food eaten. In Exp. 2, males that were fed ad libitum consumed more food and gained more body weight when females were present compared with those animals living alone. Presence of the female also had effects on reproductive maturation in males fed ad libitum - stimulating secretion of androgens, as well as growth of androgen-sensitive target tissues. However, in animals fed a restricted diet which did not allow body growth, presence of a female had little to no effect on reproduction. These results do not support our original hypothesis, but suggest that the stimulatory effects of an adult female cannot override the inhibitory effects of this level of food restriction. 
It is important to note that the effect of social cues and food availability on body weight cannot completely account for the response of the reproductive organs because the data analysis factoredout influences of body weight on the weights of these tissues (see 'Materials and Methods'). This suggests that body growth and reproductive development were affected independently by the treatments. Nevertheless, body growth and reproduction may be intertwined in an indirect way. In Exp. 2, we showed that social-cue effects were only evident in those males that were allowed to gain weight. This result can be interpreted in two ways. The presence of a female may have affected reproductive maturation through changes in energy balance (ratio of net energy cost:energy gain). By food-restricting the males, energy availability may have been at such a low level that stimulatory effects of the female could not be expressed. Alternatively, energy availability may have acted permissively for the effects of social cues on reproduction. We do not know whether a more mildly restrictive diet, perhaps to the level of males on ad-libitum feeding housed alone, would have allowed the effects of social cues to be seen.

The body composition data from Exp. 2 showed that the effects of food availability and social cues on body weight can be accounted for by alterations in total body content of water, lipid and FFDW. The weights of these 3 components were proportional to body weight, resulting in no differences in the percentages of water, lipid and FFDW to carcass weight. We have assumed that the positive effects of the female on body growth were a direct result of increased food consumption. Rissman et al. (1990) have shown that the female can stimulate growth and reproduction through a wire-mesh barrier, but this response does not occur if she is separated from the male by an opaque barrier. This suggests that one component of the female-associated cue is, at least in part, visually related. However, it is possible that other signals (e.g. tactile, localized pheromonal, localized auditory) may also play a role. The feeding stimulus is most probably specific to cohabitation with an adult female because previous work has shown that cohabitation with an adult male has no effect on body growth or sexual maturation in juvenile males compared with those males living alone (Rissman et al., 1990). At this time, we do not know why presence of the female stimulates feeding.

The effects of food restriction on reproductive maturation in other species is varied. Certainly, it can be expected that undernutrition in females would have the ubiquitous effect of delaying onset of puberty since the energy cost of pregnancy and lactation is high. On the other hand, fertility in males is not necessarily energy expensive. Rather, the energetic cost of reproductive success in the male is largely determined by his behaviour (e.g. ability to defend or to win a territory, ability to attract a female; Bronson, 1985). Hamilton \& Bronson (1985) found that food restricting wild house mice so that they were maintained near weaning weight had a differential effect on the two sexes. Food-restricted females showed a dramatic inhibition of sexual maturation compared to those females fed ad libitum, whereas food-restricted males attained a level of reproductive maturation equivalent to that of males fed ad libitum. Nevertheless, in other species, sexual maturation in the male is clearly inhibited by food restriction. In addition to the male musk shrew, the male laboratory rat (Hamilton \& Bronson, 1986; Sisk \& Bronson, 1986) and the male laboratory mouse (Hamilton \& Bronson, 1986) are reproductively suppressed if they are not allowed to gain much weight after weaning.

The present study did not address the underlying mechanisms by which food restriction suppresses reproductive development. Correlations between body weight and reproductive activity have been widely noted (Frisch, 1984; Bronson, 1985; Foster et al., 1985), and one can speculate that body weight determines the reproductive state of an animal. However, recent studies of hamsters (Schneider \& Wade, 1989) and lambs (Foster et al., 1989) suggest that changes in food intake can alter reproductive function through changes in the metabolic status of the animal, and that body weight and fat content are merely reflections of metabolic state rather than a determinant of reproductive activity. Whether the effects of food restriction on metabolic state and reproductive development are due to stress remains to be determined. Our studies in the male musk shrew suggest that food availability is an important environmental regulator of reproductive maturation. Further 
work is needed to determine whether food intake influences sexual development through changes in the metabolic state of this tropical insectivore.

We thank Jay Alexander, Marjorie Fox, Susan Harlow and Eva Lacy for technical assistance. This study was supported by NIH grant 5T32-MH-18411 (N.L.W.), NSF grant BNS-8706770 and NIH grant MH-45495 (E.F.R.), and NIH grants NS-10873, DK-32976 and MH-00321 (G.N.W.).

\section{References}

Barbehenn, K.R. (1962) The house shrew on Guam. In Pacific Island Rat Ecology: Report of a Study Made on Ponape and Adjacent Islands, pp. 247-256. Ed. T. I. Storer. Bernice P. Bishop Museum Bulletin 225, Honolulu, Hawaii.

Bartness, T.J. \& Wade, G.N. (1984) Photoperiodic control of body weight and energy metabolism in Syrian Hamsters (Mesocricetus auratus): role of pineal gland, melatonin, gonads, and diet. Endocrinology $114,492-498$.

Beg, M.A., Kauser, S., Hassan, M.M. \& Khan, A.A. (1986) Some demographic and reproductive parameters of the house shrew in Punjab (Pakistan). Pakistan J. Zool. 18, 201-208.

Bronson, F.H. (1985) Mammalian reproduction: an ecological perspective. Biol. Reprod. 32, 1-26.

Bronson, F.H. (1989) Mammalian Reproductive Biology, pp. 44-77, 175-177. University of Chicago Press, Chicago.

Foster, D.L., Olster, D.H. \& Yellon, S.M. (1985) Neuroendocrine regulation of puberty by nutrition and photoperiod. In Adolescence in Females, pp. 1-21. Eds S. Venturoli, C. Flamigni \& J. R. Givens. Year Book Medical Publ., Chicago.

Foster, D.L., Ebling, F.J.P., Vannerson, L.A., Suttie, J.M., Landefeld, T.D., Padmanabhan V., Micka, A.F., Bucholtz, D.C., Wood, R.I. \& Fenner, D.E. (1989) Toward an understanding of interfaces between nutrition and reproduction: the growth-restricted lamb as a model. In The Menstrual Cycle and Its Disorders: Influences of Nutrition, Exercise and Neurotransmitters, pp. 50-65. Eds K. M. Pirke, W. Wuttke \& U. Schweiger. Springer-Verlag, New York.

Frisch, R.E. (1984) Body fat, puberty and fertility. Biol. Rev. 59, 161-188.

Gay, V.L. \& Kerlan, J.T. (1978) Serum LH and FSH following passive immunization against circulating testosterone in the intact male rat and in orchidectom- ized rats bearing subcutaneous silastic implants of testosterone. Arch. Androl. 1, 239-240.

Hamilton, G.D. \& Bronson, F.H. (1985) Food restriction and reproductive development in wild house mice. Biol. Reprod. 32, 773-778.

Hamilton, G.D. \& Bronson, F.H. (1986) Food restriction and reproductive development: male and female mice and male rats. Am. J. Physiol. 250, R370-R376.

Harrison, J.L. (1955) Data on the reproduction of some Malayan mammals. Proc. zool. Soc. Lond. 125, 445-460.

Kirton, K.T., Desjardins, C. \& Hafs, H.D. (1967) Distribution of sperm in male rabbits after various ejaculation frequencies. Anat. Rec. 158, 287-292.

Louch, C.D., Ghosh, A.K. \& Pal, B.C. (1966) Seasonal changes in weight and reproductive activity of Suncus murinus in West Bengal, India. J. Mammal. 47, 73-78.

Rissman, E.F., Nelson, R.J., Blank, J.L. \& Bronson, F.H. (1987) Reproductive response of a tropical mammal, the musk shrew (Suncus murinus), to photoperiod. $J$. Reprod. Fert. 81, 563-566.

Rissman, E.F., Taymans, S.E. \& Wayne, N.L. (1990) Social cues influence growth and sexual maturation of the male musk shrew (Suncus murinus). J. Reprod. Fert. 89, 697-706.

Schneider, J.E. \& Wade, G.N. (1989) Availability of metabolic fuels controls estrous cyclicity of Syrian hamsters. Science, $N Y$ 244, 1326-1328.

Sisk, C.L. \& Bronson, F.H. (1986) Effects of food restriction and restoration on gonadotropin and growth hormone secretion in immature male rats. Biol. Reprod. 35, 554-561.

Wayne, N.L. \& Rissman, E.F. (1990) Effects of photoperiod and social variables on reproduction and growth in the male musk shrew (Suncus murinus). $J$. Reprod Fert. 89, 707-715.

Received 26 June 1990 